Rabaska

Revue d'ethnologie de l'Amérique française

\title{
Henri Létourneau
}

\section{Gilles Lesage et Diane Boyd}

Volume 4, 2006

URI : https://id.erudit.org/iderudit/201763ar

DOI : https://doi.org/10.7202/201763ar

Aller au sommaire du numéro

Éditeur(s)

Société québécoise d'ethnologie

ISSN

1703-7433 (imprimé)

1916-7350 (numérique)

Découvrir la revue

Citer ce document

Lesage, G. \& Boyd, D. (2006). Henri Létourneau. Rabaska, 4, 75-88.

https://doi.org/10.7202/201763ar

Ce document est protégé par la loi sur le droit d'auteur. L'utilisation des services d'Érudit (y compris la reproduction) est assujettie à sa politique d'utilisation que vous pouvez consulter en ligne.

https://apropos.erudit.org/fr/usagers/politique-dutilisation/
Cet article est diffusé et préservé par Érudit.

Érudit est un consortium interuniversitaire sans but lucratif composé de l'Université de Montréal, l'Université Laval et l'Université du Québec à Montréal. Il a pour mission la promotion et la valorisation de la recherche. https://www.erudit.org/fr/ 


\section{Portrait}

Henri Létourneau

Préparé par Gilles Lesage et Diane Boyd

Société historique de Saint-Boniface

\section{Notice biographique}

Chroniqueur, folkloriste et conservateur, Henri Létourneau est né le 13 septembre 1907 à Sainte-Delphine, Saskatchewan. C'était l'année que ses parents, Domina-Alexandre Létourneau, ferblantier de métier, et Marie-Alvina Chouinard, quittaient Saint-Eustache, Manitoba, pour s'établir à Sainte-Delphine en Saskatchewan, endroit qui avait été connu sous le vocable Montagne-la-Lime. Ils s'y étaient rendus parce que la construction d'une ligne du Grand Tronc Pacifique offrait l'occasion de se trouver de

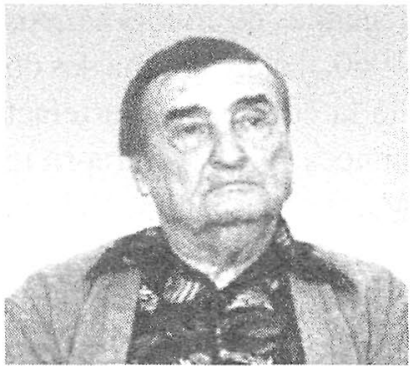
l'emploi. En effet, sitôt rendu, Domina-Alexandre était embauché comme responsable d'une pompe à eau activée par un moteur à vapeur.

- Deux ans après leur arrivée à Sainte-Delphine, les Létourneau partaient pour Indian-Head, Saskatchewan. Puis, c'est à Wolseley qu'ils s'installent avant de se rendre à Willow-Bunch. C'est à Willow-Bunch qu'Henri Létourneau entend pour la première fois les vieux chasseurs de bisons raconter leurs histoires. En 1911, la famille Létourneau retourne à Saint-Eustache où le grand-père Létourneau donne la charge de l'épicerie, comprenant un bureau de poste et un atelier de ferblantier, à son fils Domina-Alexandre. En 1914, le commerce est détruit par les flammes et la famille déménage à Thibaultville (Richer), Manitoba. En 1916, c'est à Saint-Jean-Baptiste que la famille emménage. Puis à l'automne de 1917, les Létourneau déménagent de nouveau à Saint-Boniface. Suite à la grève de 1919, la famille retourne à SaintEustache.

En 1921, à l'âge de quatorze ans, Henri Létourneau quitte l'école. Héritant de son père l'esprit d'aventure, le goût du voyage et de l'imprévu, il travailla à plusieurs endroits dans les provinces de l'Ouest : sur des fermes à la récolte des foins ou aux battages, à la construction de routes, à peinturer des églises et des maisons, et sur un ranch en Saskatchewan. De 1931 à 1937, la recherche d'emploi est diffícile et Henri Létourneau se déplace constamment. En 1937, 
il retourne d'abord à Saint-Eustache, Manitoba, où il s'adonne au théâtre et aux sports (hockey et jeu de balle). L'été, il travaille au chantier. De 1937 à 1940, on le retrouve homme à tout faire dans un camp d'été, gérant d'une salle de billard et de pompes à essence. En 1940, il travaille à l'aéroport de Carberry. Cette année-là, il épouse Rose Leclerc à Brandon. Puis en 1941, son épouse et lui élisent domicile à Saint-Boniface. Il travaille d'abord à la manufacture de Cordite et, de 1946 à 1967, il est à l'embauche de la crèmerie de Saint-Boniface. C'est en janvier 1968 qu'il commence à travailler au Musée de Saint-Boniface. Il prend sa retraite en 1985. Henri Létourneau et Rose Leclerc eurent quatre enfants, deux garçons et deux filles.

Fort de ces expériences diverses au contact d'une multitude de gens, c'est à partir des années 1950 qu'il prend davantage intérêt au patrimoine métis et canadien-français et qu'il commence à recueillir des chants et des légendes du folklore métis et canadien-français de l'Ouest. Dans les années 1960, il collectionna des objets métis et contribua largement au développement de la collection d'artefacts métis du Musée de Saint-Boniface. Durant les années 1970, il entreprit un projet d'enregistrement du folklore métis pour le Musée National de l'Homme (aujourd'hui Musée canadien des civilisations, Hull). Plus tard, la Fondation CKSB lui octroya une subvention pour faire la transcription des bandes sonores. En 1978, il publia Henri Létourneau raconte aux Éditions Bois-Brûlés suivi d'une édition révisée en 1980 et d'une nouvelle version en 1992.

La collection d'enregistrements sonores qu'il avait constituée avait été déposée au Musée de Saint-Boniface. En 1987, le Musée de Saint-Boniface transfère la collection à la Société historique de Saint-Boniface constituant le fonds Henri-Létourneau. Ce fonds documente le folklore métis et canadienfrançais du Manitoba et de la Saskatchewan. On y trouve des légendes, de nombreuses chansons, de la musique, la description de jeux d'enfants, des recettes, des témoignages divers, des récits d'événements importants du passé, des descriptions de métiers et des renseignements biographiques. Il a interrogé des gens des villages suivants : Saint-François-Xavier, Baie-Saint-Paul, Piapot, Duck-Lake, Batoche, Portage-La-Prairie, Saint-Boniface, Richer, Skull-Creek, Eastend, Saint-Labre, Woodridge, Rosthern, Sainte-Rose-duLac, Cayer, Saint-Norbert, Belcourt (Dakota du Nord), Saint-Eustache, SaintLazare, Biscarth, Saint-Joseph, San-Clara, Saint-Georges, Saint-Pierre, SaintVital, Saint-Charles, Saint-Malo, Otterburne, Saint-Laurent, Sainte-Annedes-Chênes. Le fonds comporte aussi des entrevues avec Henri Létourneau.

Son engouement pour la chose folklorique est devenu légendaire. Comme il l'écrit en quatrième de couverture dans Henri Létourneau raconte, publié aux Éditions Létourneau (Éditions G.F.L.), 1992 : 
Les histoires des Anciens ont toujours eu pour moi un attrait tout particulier. C'est la petite histoire du Manitoba où dorment sous le " gumbo » quatre générations de ma famille, et celle de la Saskatchewan où je suis né.

Ce pays à moi, c'est l'Ouest; pays d'adoption de mes grands-parents paternels et maternels ; l'Ouest où sont nés mes parents, mes enfants et mes petits-enfants. Ces plaines immenses qui semblent en flammes au lev[er] du soleil ! Ce soleil qui glisse dans une mer rouge quand il se couche ! Ces lev[er]s et ces couch[er]s du soleil sont à moi !

L'aurore boréale qui danse au-dessus de ma tête... Le firmament couvert d'étoiles scintillantes... La brise chaude et caressante du printemps... Le cri de l'outarde qui, le printemps, arrive du sud, et à l'automne, y retourne....

Toutes ces choses sont à moi !

Henri Létourneau est décédé le 28 novembre 2002 au Centre hospitalier Taché à Saint-Boniface, Manitoba. 


\section{Entrevue avec Henri Létourneau ${ }^{1}$}

\section{Origines de sa famille}

Du côté maternel, mes grands-parents venaient du Michigan, aux États-Unis. C'était des immigrés qui avaient parti de Saint-Jean-Port-Joli, pour s'en aller demeurer au Michigan alors chercher du travail comme de raison. Après quelques années au Michigan, mes grands-parents sont en venus à SaintPierre, ici. Je crois qu'il y a tout un groupe qui est arrivé en 1877. Les Chouinard, les Archambault, les Deault, dont ils donnaient leur nom Dean : ç'avait changé aux États-Unis à Dean. Et puis je pense, bien, ils étaient tous parents, ces gens-là, quoique mon grand-père maternel avait marié une Archambault, rendu à Saint-Pierre ici. Alors, il a eu seulement que des filles, je crois que c'est cinq filles. Et, donc ma mère en était une. Alors ma mère était orpheline toute jeune et elle s'est en venue ici. Ils l'ont mise à l'orphelinat, à Saint-Boniface, à l'âge de neuf ans. Elle était ici quand Monseigneur Taché est mort. Et elle se souvenait très bien aussi de la sœur indienne, c'est-à-dire de la tribu des Sioux qu'il y avait ici, les premières sœurs Grises, sœur Nebraska. Elle en a parlé souvent et que les petites filles étaient toutes habillées pareil. Des jupes avec des laises. Des laises, apparemment, c'est des lisières que ça veut dire. Des laises jaunes et des laises rouges. Et puis un petit manteau, espèce de mantelet qui était jaune et rouge, et puis un petit casque qui avait des laises rouges et puis jaunes. Elle dit qu'ils avaient l'air des polichinelles, tout probable avec des costumes comme ça; mais, apparemment, c'était la mode à l'époque ici, à l'orphelinat.

Un peu plus tard, elle a été adoptée par une famille Boisvert de SainteAnne. Lui, Monsieur Boisvert venait du Québec, mais sa femme était une métisse du nom de Dumais. Et puis d'elle, on a eu beaucoup d'histoires des premiers temps. C'est ça qui m'a donné le goût, peut-être, des histoires du Manitoba des premiers temps, parce qu'elle se rappelait d'avoir été à la chasse au bison avec ses parents quand elle était petite fille en charrette, avec une vache attelée sur la charrette, plutôt d'un bœuf; alors leur famille avait du

1. Ce document est la transcription d'une entrevue qu'Henri Létourneau a donnée en 1981 à CKSB, poste de Radio-Canada à Saint-Boniface. La transcription a été préparée par le Laboratoire de littérature orale (LABOR) au Centre acadien, Université Sainte-Anne. 
lait soir et matin, du fait qu'ils avaient une vache. C'était la seule dans le camp qui pouvait avoir du lait. Alors ma mère a tout entendu ces histoires-là. Et puis, d'après sa mère adoptive, elle savait beaucoup de choses à propos des plantes qui pouvaient servir de remèdes. Elle en savait beaucoup. Elle m'en a montré beaucoup de ça, ma mère, qu'elle avait entendu par sa mère adoptive. Et puis, sur la fin de 1905, elle a marié mon père, Domina Létourneau. Lui, le grand-père Létourneau, Frédéric, venait aussi de la province de Québec, de Saint-Constant. Mais apparemment, il avait fait un apprentissage de trois ans à Laprairie au Québec, comme ferblantier. Son métier était ferblantier. Plus tard, il était au Vermont. Il aimait pas les ÉtatsUnis, alors il est revenu au Canada. Et puis, en ce temps-là, dans une partie de l'Ontario, là, tout près des lignes du Québec, il y a un endroit qui s'appelle Embrun. J'ai été visiter ça, il y a un mois, par curiosité : c'est une grosse paroisse canadienne-française. Il y a 2900 âmes à Embrun. Embrun aujourd'hui est encore français pareil comme à l'époque quoique c'est dans l'Ontario. Et puis de là, il avait rencontré une demoiselle Latrémouille dont le père était hôtelier à Hull, au Québec. Et puis il l'a mariée. Lui, il avait vingt-trois ans et puis elle avait quatorze ans. Une bonne fois, à Ottawa - il était en voyage à Ottawa - c'est pas loin de là, il rencontre un monsieur. Ils étaient deux frères - $\mathrm{j}$ 'ai oublié leurs noms - qui avaient un magasin de quincaillerie sur la rue du bureau de poste, qui est la Lombard aujourd'hui, à Winnipeg. Ils avaient absolument besoin d'un ferblantier. Alors, ils ont offert à mon grand-père la somme magnifique d'un dollar par jour. Et puis, il a pas pu résister. Il avait jamais entendu parler que c'était possible de gagner un dollar par jour ; ça vous donne une idée des salaires dans la province de Québec. Alors, il s'est en venu jusqu'à Saint-Claude, Minnesota. Puis de là, c'était en bateau sur la rivière, hein, un bateau à vapeur sur la rivière Rouge. Et puis, deux mois après, la grand-mère, avec les deux aînés des enfants qui étaient à Embrun, un petit gars puis une petite fille, elle s'est en venue, elle aussi, le retrouver ici.

Une chose assez curieuse, quand on prend l'histoire, il y a eu seulement qu'un Létourneau qui est venu au Canada en 1658. C'était un homme de métier aussi, qui était venu au Canada à la demande de Monseigneur de Laval - c'était un meunier - pour prendre soin du moulin à farine de Monseigneur Laval, qui se trouvait à être à Château-Richer. Alors, notre ancêtre Létourneau était venu tout seul, a pris soin du moulin puis, deux ans après, il a fait venir sa femme puis aussi ses deux enfants. Alors une chose qu'on peut se vanter, nous autres, les Létourneau, c'est que, du côté des Létourneau on a été payé pour venir au Canada puis payé pour venir dans l'Ouest. C'est un peu différent des autres immigrants qui sont chassés des pays d'Europe, qui sont obligés de s'en aller à cause de la misère et puis des 
guerres; et je pense que c'était assez rare. À part de ça, en 1937, ça fait déjà plusieurs années, il y avait déjà quatre générations de ma famille dans le cimetière de Saint-Eustache, hein. Alors, on peut dire qu'on est vraiment de l'Ouest maintenant, alors moi, né en Saskatchewan.

\section{Sa naissance}

Quand ma mère a rencontré mon père, un an après, ils se sont mariés. Elle, elle demeurait à Lorette. À l'époque, les Boisvert étaient déménagés à Lorette. Et puis, ils se sont mariés. Et puis, l'année d'ensuite, mon père a été travailler pour le Grand Tronc Pacifique qui était construit. À l'époque, il était rendu en Saskatchewan. Il s'en allait du côté du Pacifique. Et puis, actuellement, que leur terminal, eux autres, était à Prince-Rupert, au lieu de Vancouver. Alors, il travaillait, il avait soin d'une locomotive qui pompait de l'eau, à un endroit qui s'appelait à l'époque (c'était une petite paroisse française) SainteDelphine. Aujourd'hui, ça n'existe plus. Je sais pas quand ç'a disparu. Aujourd'hui, il y a un village tout près, en grande partie ukrainien. Les gens qui demeuraient à Sainte-Delphine, le vieux nom était la Montagne-la-Lime. Puis, il y a une partie encore qui s'appelle, en anglais, File-Hill, c'est-à-dire la Montagne-la-Lime. Et puis, c'est là je suis né, le 13 de septembre de 1907, il y a 74 ans, la semaine passée. Je me souviens pas de l'endroit du tout. On a parti, j'étais trop jeune. Mais ma mère m'a raconté souvent que, quand je suis né, mon père a parti à cheval pour aller chercher une sage-femme. Il y avait pas de médecin alentour. Et puis, il a été voir une dame qui avait du sang assiniboine, elle était en partie indienne. Elle a sauté sur son cheval, puis elle était tellement bonne - comment ce qu'on dirait ça ? je sais pas si c'est le mot français équestrienne [cavalière] -, mais en tous les cas, elle est arrivée longtemps avant lui. Elle pouvait aller beaucoup plus vite que lui. Elle était habituée à aller à cheval, bien mieux que mon père. Alors, quand mon père a arrivé, j'étais né déjà. Et puis, ma mère m'a raconté souvent que le seul jour de Noël qu'elle se souvient, c'est-à-dire la fête de Noël à SaintDelphine, la messe de minuit était dans une petite église en billots recouverts de glaise. Il y avait pas d'orgue ni d'harmonium, comme ils appelaient ça à l'époque. Il y avait deux violons, deux Métis avec des violons. Ils jouaient des airs tellement entraînants pour les cantiques que tous les gens, ils entendaient un vacarme dans l'église, tous les gens tapaient du pied. Alors, pour elle, c'était une chose vraiment curieuse. Elle avait jamais vu ça au Manitoba.

\section{Un souvenir de Willow-Bunch}

Et puis de là, bien, on est allé dans la vallée de $Q u^{\prime}$ Appelle. La vallée de Qu'Appelle, on a été à Willow-Bunch qui se trouvait dans la « Montagne de 
bois ». Ça, je me souviens un peu d'avoir vu le vieux Jean-Louis Légaré qui avait des cheveux coupés en balai, qui était très vieux à l'époque puis qui était un des pionniers de l'endroit, hein, dans le temps des Indiens, surtout des Sioux. Et puis on demeurait un peu en-dehors du village. Mes parents avaient un homestead là. Puis il y a quelque chose que peut-être que je me rappelle pas, mais ma mère l'a conté tellement souvent qu'on vient qu'on s'imagine qu'on a vu ça, vous savez quand on est petit, hein ? Parce que me semble que j'étais un peu jeune pour me souvenir de ça. Mais je sais que, apparemment, je jouais un après-midi, là, devant la maison. Et puis c'était juste quoi ce qu'à l'époque qu'ils appelaient un shack de homesteaders, des gens qui ont pris du terrain. Et puis $\mathrm{j}$ 'avais un magasin. Je me souviens très bien de mon magasin, c'était des vieilles caisses de bois, à l'époque c'était pas des boîtes en carton, c'était des boîtes de bois. Alors, je m'étais fait un comptoir et puis j'avais, dans des bouteilles, j'avais toutes sortes de rassades, comme les Métis les appelaient, de perles que les Indiens achetaient des blancs, hein. Elles étaient faciles à trouver, on en trouvait un peu partout dans l'herbe. Anciennement, les Sioux qu'il y avait là enterraient pas leurs morts, alors ils les accrochaient sur des arbres. Ils les accrochaient dans les arbres puis où [est-] ce qu'il y avait pas d'arbres, ils les mettaient sur une espèce de petite plate-forme qui, à la longue, tombait par terre. Les loups charriaient les ossements partout, alors on pouvait trouver dans l'herbe... Ma mère voulait pas les toucher parce qu'elle avait peur des morts. Mon père, ça lui faisait absolument rien puis moi j'étais trop jeune pourvu qu'ils étaient lavés, qu'ils étaient propres, c'était le principal. Alors cet après-midilà, je jouais avec ça quand tout d'un coup, j'ai vu que ma mère avait bien peur. Il y avait quelque chose qui lui faisait peur puis elle regardait la grande côte qu'il y avait en avant de la maison chez nous, c'était un pays roulé, vous savez, de collines. Alors, il y avait quelque chose qui lui faisait peur. Elle était toute seule à la maison. Ce matin-là, la police montée avait passé avec des wagons et puis des chevaux attelés dessus, puis elle ramassait tous les hommes qui étaient capables pour aller battre un feu de prairie, un immense feu de prairie, alors fallait qu'ils l'éteignent. La police montée à l'époque avait le droit de... n'importe quel homme pouvait laisser son travail puis y aller ; mon père était parti. Puis, en tous les cas, c'était quelqu'un qui descendait la côte qui lui faisait peur. Alors on a rentré dans la petite maisonnette et puis elle a poussé le verrou puis elle regardait par la fenêtre. Mais elle m'a raconté plus tard qu'elle voyait un Indien qui s'en venait avec un grand couteau, qui faisait aller son couteau, comme ça puis les reflets du soleil, elle pouvait voir les reflets métalliques. Quand il a arrivé tout près de la maisonnette, elle a poussé un soupir de soulagement puis elle a ouvert le verrou. Puis là ç'a frappé à la porte : elle a ouvert la porte. Je me souviens de 
l'avoir vu. C'était un vieil Indien tout ridé qui avait des boutons en os, là ; c'est à l'époque, des grands boutons en os qu'étaient accrochés après ses oreilles avec des fils de babiche, de cuir, qui passaient, une corde de cuir qui passait dans les bas des oreilles, elles étaient percées. Mais c'était tellement lourd, puis le bas de l'oreille, il touchait presque à l'épaule, hein. C'était étendu comme un beigne croche, là... Et puis son fameux couteau, c'était la partie supérieure de l'aile d'une oie, une oie grise, alors vis-à-vis le soleil, ça faisait des reflets métalliques, hein. Il faisait tellement chaud qu'il s'avait fait un éventail et elle pensait qu'il brandissait un couteau, hein. Quoi ce qu'il voulait, l'homme, c'était d'avoir de l'eau fraîche, alors elle lui a donné, comme qu'on dit en canadien, le diper [une écuelle], puis il s'est en allé en arrière de la maisonnette ; là, il y avait une source d'eau vive, très froide, qui sortait de dans le pied de la colline en arrière. Alors ça, c'était une des aventures. Peut-être qu'aujourd'hui c'est des choses qu'on voit pas.

\section{Souvenir d'un déménagement}

Ensuite, quand mes parents ont décidé de revenir au Manitoba, fallait s'en revenir en wagon pour prendre le train à Moose-Jaw. Quatre-vingt-cinq milles en wagon avec des chevaux, hein. Alors mon père avait parti en avant et puis j'étais avec ma mère puis mon frère qu'était bébé à l'époque, Marcel, le deuxième. Alors, je me souviens vaguement du voyage. C'était très long parce que les journées étaient pas longues. C'était ce qu'ils appelaient une brigade de wagons qui s'en venait chercher; ils emmenaient des voyageurs puis ils emmenaient des produits de la ferme pour vendre dans la ville de Moose-Jaw. Et puis c'était pour emporter toutes sortes de choses pour le magasin de l'endroit, cour à bois, il y avait tout quoi ce qu'ils avaient besoin. Alors, c'était vraiment une brigade. Ils étaient plusieurs. Et puis il y avait une tente qu'ils érigeaient tous les soirs pour moi puis, bien pour mon frère et moi puis notre mère, hein. C'était une tente avec un trou dans le haut; dans l'automne tard, ils pouvaient mettre un petit poêle puis le tuyau aurait passé par le trou, hein. Un soir qu'elle était toute seule avec nous deux et puis que mon frère pleurait, il était malade - je sais pas si c'était des coliques ou il y avait quelque chose - toujours, il pleurait. Elle avait allumé le fanal pour avoir de la lumière. Tout d'un coup, il y a une voix d'en haut qui a dit : "Baby sick ». Et puis elle regarde en l'air : c'était un Indien qui regardait par le trou, l'ouverture pour passer le tuyau, hein, en pleine nuit. Alors, elle pouvait pas s'imaginer comment un homme pouvait être si grand pour regarder par là, hein. Mais c'est seulement que, après qu'elle a eu dit « yes », là, le visage a disparu ; puis là, on a entendu un cheval qui s'en allait, le pas d'un cheval, alors c'est tout probable qu'il était debout sur son cheval pour être capable de regarder. Vous savez que la lumière dans la tente, c'est tout probable qu'il 
voyait des ombrages de ma mère, là, qui devait marcher alentour pour essayer - peut-être qu'elle portait le bébé dans ses bras - pour essayer de l'endormir, je le sais pas. En tous les cas, ça c'était le voyage. Quatre-vingt-cinq milles. Je sais pas combien de jours que ç'a pris, mais je sais qu'on avait passé un endroit que tout était noir, une grande côte qu'était toute noire, puis il y avait des ronds blancs dedans. Alors, plus tard, ma mère $m$ 'a dit que ces ronds blancs-là, c'était un endroit où ce que le feu avait passé : toute l'herbe avait brûlé. Puis les ronds blancs, c'était un grand village indien qu'il y avait là, il y a bien longtemps, alors en brûlant l'herbe, on voyait seulement que les pierres qu'ils mettaient à la base de leurs tipis. Les tipis étaient ronds, alors vous aviez un rond fait avec des pierres blanches : c'était comme tout des zéros blancs, comme sur une ardoise noire, là, qui avait été dessinée.

\section{Le jeu des osselets}

Très jeune, vous savez, nous autres c'était une famille qui était pas comme les autres. Mon père c'était une espèce de Juif errant. Il se plaisait pas nulle part, alors au bout d'un an ou deux, fallait déménager. Et puis il avait tout un système. Il partait le premier, puis ensuite il écrivait à ma mère ; ma mère était obligée de tout empaqueter elle-même, puis elle était prise avec les enfants, puis déménager, hein, toute seule. Alors, lui se sauvait de ce travaillà. C'était vraiment une maladie pour lui, ça, de déménager. J'ai été dans plusieurs écoles au Manitoba. Et puis, comme par exemple, quand on était jeune, bien c'était les jeux de l'époque. Avant qu'on soit assez vieux pour jouer à la balle, bien, c'était jouer aux osselets. C'est quelque chose qu'est oubliée maintenant. Seulement que les gens se faisaient leurs propres osselets. Osselets veut dire des petits os, hein. Alors, quand ils faisaient boucherie, quand ils tuaient surtout un cochon, là, ils prenaient les petits os, les ergots. Après qu'ils avaient été bouillis, là, la chair s'enlevait toute. C'était toute une forme particulière. C'arrivait comme une petite jointure. Alors ils prenaient tout ça, ils les recueillaient et puis là, ils donnaient ça aux enfants. Puis ils leur disaient d'en avoir bien soin, c'était des osselets, ils pouvaient jouer aux osselets. Ils pouvaient se servir de ça, ouais.

\section{Aller à l'école sans chaussures}

Les temps ont toujours été durs, pour la raison [que] ça dépendait où on a demeuré. Où [est-]ce que c'est qu'on a fait une grande bêtise, c'est quand on a été demeurer à Thibaultville à l'époque, ce qu'ils appellent Richer aujourd'hui. C'était très pauvre. Les gens se prenaient des terres là, et puis ma mère avait deux de ses sœurs mariées qui demeuraient à Richer. Alors eux autres vantaient le terrain. C'était des gens qui venaient du Québec, les deux frères, là, qui avaient marié les deux sœurs, mes deux tantes. Et puis 
pour eux, au Québec, les terres qui avaient de la roche, c'était des terres fortes. Alors ils ont été se fourrer dans la roche par-dessus la tête. Puis vraiment c'était un cas incroyable. Quand que mon père a vu le terrain de Richer, là ses beaux-frères aimaient qu'il prenne du terrain là -, il a refusé complètement parce que, comparé à Saint-Eustache qu'était à peu près les terres les plus riches du Manitoba, il y avait pas de comparaison entre les deux places. On a passé quand même deux ans là. C'est là que j'ai vu une chose assez curieuse. Les enfants étaient pas riches, alors c'est le seul endroit au Manitoba (j'ai été dans plusieurs écoles au Manitoba) que j'ai vu les enfants à l'école nu-pieds l'été. Ils étaient nu-pieds, hein. C'était à Richer, et je l'ai jamais vu ailleurs. À l'époque, Thibaultville puis c'était encore, je pense le nom vraiment devrait être Thibaultville. C'est le bureau de poste qui s'appelait Richer, même à l'époque, mais les gens disaient toujours Thibaultville.

Et puis de là, on est revenu... Oh! je devrais peut-être conter la mort d'un de mes petits cousins, là. Quand c'est venu pour aller à l'école après les vacances, il aurait bien voulu aller à l'école, mais il était trop jeune. Seulement qu'il aurait voulu avoir des bottines puis il en avait pas. Et puis on disait : « Tu vas pas à l'école cette année, tu auras pas de bottines. L'année prochaine ». L'argent était trop rare, hein. La vie était très dure à Thibaultville. Alors, dans l'automne tard, le petit, quand toute la famille était à l'étable à traire les vaches, il courait sur la gelée, dehors, nu-pieds, hein. Et puis, il a viré à l'étable. Tout d'un coup, il a attrapé le croupe. Et puis le croupe à l'époque, ça pardonnait pas, hein. Ils avaient pas des médicaments pour. Il y avait des bons docteurs, mais ils pouvaient pas, à moins des fois, les percer dans la gorge pour les empêcher d'étouffer, ils leur faisaient des trous dans la gorge pour qu'ils puissent respirer. D'habitude, c'était la mort, hein. Alors lui avait tout près de six ans, puis une petite tête noire, là, toute frisée. Il était très joli, puis je me souviens qu'il était malade, là, dans son petit lit, là. Je me souviens quand il a étouffé, quand il est mort, là. Sa mère était debout puis elle a parti à pleurer, puis elle a jeté la couverture après sur sa tête. C'est la première fois que je voyais quelqu'un mourir, hein. Et puis, son père quand il a revenu du travail, là, dans les bois (il bûchait du bois), il dit : « Il voulait avoir avec des bottines ", c'était un grand désir ça depuis qu'il était tout petit, avoir des bottines, puis il en avait pas. Alors il dit : « Il va en avoir. Il va être enterré avec des bottines ». Puis ma tante a dit : « [ $\grave{A}]$ quoi ça sert ? C'est rien, il dit, il voulait avoir des bottines puis il va les avoir. » Alors, il a été dans le bois le lendemain matin, très de bonne heure. Il a bûché jusqu'au midi, une charge de bois, puis il a attelé ses chevaux là-dessus, puis il a transporté ce bois-là à Sainte-Anne, où ce qu'il pouvait vendre son bois, hein. Et puis le soir, il est revenu avec une paire de bottines et puis le petit a 
été enterré avec des bottines. Alors, ça vous donne une idée pas mal, de l'attitude des gens et la misère, hein. La grande misère.

\section{L'épluchette de blé d'Inde manquée}

Le seul endroit que j'ai vu une épluchette de blé d'Inde, à part de celle de dimanche passé, là, ici à Saint-Boniface, c'était à Richer. Mon oncle avait fait une épluchette de blé d'Inde, puis apparemment c'était l'hiver, parce que c'était en hiver qu'il avait fait ça. Alors, il avait mis un gros tas de blé d'Inde sur une couverture de lit dans le milieu de la place, puis on était tous assis alentour, hein. Et puis là, il nous a dit qu'il était pour nous conter un conte : "La princesse blanche ». Alors ses enfants étaient tout fiers, mes cousins puis cousines. « Ça fait bien longtemps que vous l'avez pas conté, puis elle est tellement belle, cette histoire-là. » Alors, il s'est assis puis il a commencé : « Là, vous savez, il y avait un roi qui avait une fille qu'ils appelaient la princesse blanche. » Là, ç'a frappé à la porte. C'était le soir, hein. Il faisait noir plus tôt en hiver. Alors il a ouvert la porte, et puis c'était deux gros messieurs en capot de fourrure. Ils ont donné la main puis ils ont dit qu'ils travaillaient à des élections. C'était des cabaleurs, hein. Ils passaient d'une porte à l'autre. Puis apparemment, il était pour [y] avoir des élections. Et puis, ils faisaient boire un verre au propriétaire, là. Comme de raison, mon oncle a tout oublié l'épluchette de blé d'Inde, et puis ma tante a tout serré le blé d'Inde, a tout enlevé de sur la place. On a été se coucher, puis ils en ont jamais refait d'autres, alors on peut appeler ça « l'épluchette de blé d'Inde manquée $»$. Et puis la fameuse histoire, j'aurais bien aimé l'entendre. Je l'ai jamais sue. Il l'a jamais racontée après.

\section{Comptines}

Et puis de là, on est revenu à Saint-Eustache, et puis de Saint-Eustache, bien, les jeux encore, j'étais un peu jeune. C'était mon petit jeu, vous savez, "Sur le pont d'Avignon ». Vous avez entendu ça déjà ? Et puis les vieux, ce qu'ils appellent des complaintes [comptines] je pense au Québec. Par exemple : " Ma tante Michelle, un i un 1 », "Un loup passant par le désert lâche un hum [pet] pour qui ? pour toi ». Puis celui que, c'est pour lui, c'est lui qui est le loup ou bien non qui est [éliminé ou choisi]... Et toutes sortes de choses comme ça. Et puis, oh, il y en a plusieurs.

J'en ai fait toute une liste, là, dans mes enregistrements. Quand on est arrêté puis qu'on peut y penser, ça nous vient à la mémoire. « Pom Pom par là oui ». Finalement, là, on a été à Saint-Jean-Baptiste. À Saint-Jean Baptiste, pour moi, là, ça valait vraiment quelque chose. J'aurais dû dire, par exemple, que $\mathrm{j}$ 'ai fait ma petite communion, la première communion qu'ils appelaient à l'époque, à Richer, ma confirmation à Saint-Jean-Baptiste, ma communion 
solennelle à Saint-Eustache et puis, finalement, de Saint-Eustache, on est revenu, on était à Saint-Boniface à la fin de la guerre, la Grande Guerre de 1919. On était ici. C'est là que mon père a décidé de retourner à SaintEustache. Alors, on est retourné à Saint-Eustache. Et puis là, on a demeuré à Saint-Eustache jusqu'à quand mes parents se sont retirés, vous savez. Bien moi, ça fait 41 ans que je suis à Saint-Boniface.

\section{Relations avec les anglophones}

Mais les grands ranchs de la Saskatchewan, sur les frontières de l'Alberta, un peu partout, hein, là, j'ai tout connu ça. Et puis c'est surtout travailler, j'ai travaillé beaucoup chez les Anglais, des places même complètement anglaises, et puis, s'il y avait des Français alentour, on parlait en français, mais j'ai jamais entendu dire quoi ce qu'il y a des gens qui disent des fois, là : « Speak white ». J'ai jamais entendu ça. Du moins, jamais, personne m'a jamais dit, alors j'ai même pas entendu dire [ça] à d'autres, j'ai été dans des endroits que c'était complètement anglais. Par exemple, il y avait un grand riche, Seventy-six, près de Maple-Creek, sur les frontières d'Alberta. On était un été jusqu'à vingt-cinq hommes puis, là-dessus, il y en avait peut-être dix à douze qui étaient canadiens puis métis, de langue française, et puis on n'a jamais eu de trouble. On parlait tout français entre nous autres, puis quand était à parler aux autres, on leur parlait en anglais, comme de raison. On n'a jamais eu de trouble du tout, alors pour moi, c'est quelque chose ne nouveau. Même hier, il y a un monsieur qui m'a dit que ça lui a été dit déjà. Mais moi, c'est quelque chose que j'ai jamais entendu ça, hein.

\section{La Dépression}

Pour moi, là, avoir travaillé un peu partout comme ça, vous savez, j'ai été obligé d'abandonner l'école à l'âge de 14 ans, seulement qu'au grade huit. Alors, j'aurais aimé avoir été au collège. Ça m'aurait aidé beaucoup aujourd'hui, mais les expériences que j'ai eues puis les choses que j'ai vues, ça, ça s'achète pas avec aucun argent, hein. Si j'avais été au collège, peutêtre que j'aurais jamais vu ça. Alors pour moi, c'est une expérience que j'ai pas de regret du tout. Même pendant la Dépression, c'était très dur, hein. L'ouvrage était très rare. J'ai travaillé pour cinquante sous par jour, dix heures par jour. Ça veut dire cinq sous de l'heure, hein. Mais j'oublierai jamais ça parce que j'ai jamais tant joué à la baseball l'été et puis au hockey l'hiver comme pendant la Dépression, les dix ans de la Dépression, hein. C'était incroyable, on avait du temps pour jouer. Quoique, la moitié du temps, on avait des bien vilains patins, nos sticks, on était obligé de les réparer nous autres mêmes, nos bâtons, parce qu'on n'avait pas d'argent pour en acheter d'autre ; et puis, à part de ça, quand on jouait à la baseball, bien souvent, on 
n'avait pas même de gants, hein. Ceux qui étaient dans le champ [n']avaient pas de gants, là. Ils prenaient la balle dure nu-mains, là. C'était fort, assez dur. Et puis celui qui était receveur, en arrière du bat, là, bien souvent, [n']avait pas même de masque ou de protecteur ; alors c'était pas mal dur. Mais, quand j'y pense aujourd'hui, à ce temps-là, me semble [que] ça valait la peine. Tout le monde était égal. Tout le monde était pauvre. Il y avait personne de riche. Même des gros fermiers [n']étaient pas mieux que celui qui travaillait à gage. Peut-être qu'il avait aucune inquiétude pour la nourriture, mais quand même, ses affaires, elles allaient bien mal, hein, parce que son grain se vendait pas.

\section{La retraite}

Si je me retirais complètement, je pourrais écrire plus à mon aise parce que, là, j'ai un deuxième livre qui va être prêt, bientôt, en français, des histoires que j'ai recueillies d'un bord et de l'autre. Et puis depuis des années que les gens me téléphonent, même ceux qui comprennent pas le français, des fois, lisent mes livres avec un dictionnaire, puis c'est pas fameux ça. Alors, ils me demandent d'en écrire un en anglais qui serait basé sur un des autres. Alors, c'est tout probable que je vas en sortir un pendant l'hiver, hein. On n'est jamais trop vieux.

Quand j'avais 65 ans, j'aimais pas la danse. J'haïssais la danse. Alors j'ai pensé, vu que ma femme aimait bien la danse, et puis quand on allait en quelque part qu'il y avait une danse, elle me traînait sur le plancher, hein. Elle voulait absolument danser, puis j'aimais pas ça. J'ai pensé : je vas prendre des leçons de danse, hein. Alors, j'ai pris des leçons de danse, je l'ai pas dit à ma femme. Puis une bonne fois, on était à une grande veillée puis je l'ai demandée [à] danser. Elle était un peu surprise, car c'était moi qui la demandais. C'était une valse et puis elle dit : «Tu peux pas danser avec moi. » C'est là que j'ai appris que c'est presque impossible pour un qui a jamais dansé avant puis qui a pris des leçons pour danser avec eux, parce que lui fait les vrais pas, puis les autres le font pas, hein. Alors, il y a pas d'accord entre les deux. Moi, je pouvais pas comprendre comment ça se faisait qu'elle pouvait pas danser avec moi. Alors, elle dit : "Quoi c'est que ça veut dire, ça ? Où [est-]ce t'as appris ça, essayer de faire danser de même ? » Là a fallu que j'y aie dit, puis elle a pris des leçons elle aussi. Alors maintenant quand on va à une danse, on peut danser ensemble. Là, on danse le vrai fox-trot ou la vraie valse, la grande valse ; puis bien mieux que ça, il a été un temps, avant que j'aie mal aux genoux, le rhumatisme, on dansait le cha-cha puis la samba, puis la rumba, nous autres, on a tout appris ça, hein. Alors ma femme était très bonne. Elle a toute une grand-table toute couverte de trophées, hein, qu'elle a gagnés... Aujourd'hui, elle y va plus, elle prend plus de leçons. 
Mais c'est juste pour dire, là, c'était un désennui. Alors, il y a toujours quelque chose qu'on peut faire, hein.

Quand même, à mon âge, là, il y en a, je vois de mes amis qui se sont retirés aussitôt qu'ils ont eu 65 ans. Ils sont plus ici aujourd'hui, ils sont partis, parce que c'était des gens qui avaient rien pour s'occuper. La femme, elle, quand elle se retire, elle a toujours son même petit travail à faire : le soin de la maison. L'homme, lui, il fume sa pipe ou bien, s'il fume pas, il se promène les mains derrière le dos sur les trottoirs de la ville. Et puis la première chose qu'on sait : un tel est parti, hein. Alors, il y a pas rien pour les tenir occupés, tandis que même l'affaire de faire le ménage pour la femme, là, c'est pour ça qu'aujourd'hui, la femme vit beaucoup plus longtemps que l'homme, c'est parce qu'elle a quelque chose, hein. Alors, $j$ 'ai pensé, avant de partir je suis aussi bien de faire ma marque, vraiment faire quelque chose. 